es auf der Kurve $A(R)$ nach links zu kleineren Werten von $A$ und damit von $\theta$ gelangt. Nach Kornetzki ist bei $\mathrm{Fe} \Delta \theta / \Delta p-5$ bis $-10 \cdot 10^{-3}{ }^{0} \mathrm{C} / \mathrm{at}^{6}$. Nach Slater, De Boer und Michels ist für Ni $\Delta \theta \Delta p+0,0510^{-3}$ bzw. $+0,00810^{-3}{ }^{0} \mathrm{C} /$ at $^{7}$.

Um das entgegengesetzte Verhalten von Ni zu erklären, nimmt man seine Lage rechts vom Maximum von $A(R)$ an. Abstandsverkleinerung läßt dann $A$ und damit $\theta$ wachsen. Auch hierfür ist eine theoretische Begründung bisher nicht gegeben; man nahm früher sogar Ni bisweilen links vom Maximum von $A(R)$ an.

Eine Berechnung von $A(R)$ durch den Verfasser mit dem Radialanteil der $3 d$-Funktionen ergab für $A$ ständig negative Werte, wie dies allgemein in der Valenztheorie angenommen wird. Weiterhin scheiterten Versuche, das verschiedenartige Verhalten von $\alpha$ - und $\gamma$-Fe durch besondere Formen einer positiv werdenden Austauschintegralfunktion verständlich zu machen. Die in ${ }^{4}$ dargelegte Vorstellung ist andererseits in der Terminologie des Heitler-London-Modells gesprochen nur mit einem ständig negativen $A(R)$ verträglich.

Im folgenden soll gezeigt werden, wie das Vorzeichen der Druckabhängigkeit der Curie-Temperatur $\theta$ bei Fe und $\mathrm{Ni}$ richtig aus der nunmehrigen Theorie ${ }^{4}$ verständlich gemacht werden kann.

Bei Abstandsverkleinerung heben sich die Fermigrenzen der lockernden Bänder bei Ni und besonders bei $\mathrm{Fe}$ weiter über das Atomniveau wegen der größer werdenden Wechselwirkung der Atome untereinander. Da das $s$-Band mit 0,6 bis 0,8 Elektronen pro Atom nur zu $30-40 \%$ gefüllt ist, wird sich in ihm bei seiner Verbreiterung die ursprüngliche Fermi-Grenze etwas senken, so daß Elektronen von den gehobenen oberen Bändern in dieses $s$-Band übergehen, während die Elektronenzahl in den aufgefüllten unteren konstant bleiben muß.

Links vom Maximum der Slater-Kurve (Fe) verlieren die oberen Bänder Plus-Spins, da in ihnen noch keine Minus-Spins enthalten sind, so daß die Sättigungs- magnetisierung und damit $\theta$ abnimmt. Rechts vom Maximum werden aber die zuletzt eingetretenen Minus-Spins zunächst abgehen, so daß sich die Magnetisierung und damit $\theta$ erhöht. Der hier benutzte Zusammenhang zwischen Sättigungsmagnetisierung und $\mathrm{Cu}-$ rie-Temperatur wird durch die in ${ }^{4}$ entwickelte Vorstellung in guter Übereinstimmung mit der Erfahrung geliefert. Da die Bandausweitung unter Druck außerdem die Fermi-Energie erhöht und damit $\theta$ erniedrigt, wird der obige Einfluß des Druckes bei Fe verstärkt, bei Ni aber abgeschwächt.

Die hier angenommene Änderung der Sättigungsmagnetisierung durch Druck ist im vorliegenden Sinne experimentell für $\mathrm{Ni}$ bei höheren Temperaturen und vor allem für Fe bestätigt, während bei Ni für tiefere Temperaturen sich widersprechende Ausdeutungen der Messungen vorliegen ${ }^{8}$.

Néel trug über dem Abstand der $d$-Schalen den gemessenen Faktor $N$ des Weisschen Molekularfeldes auf und erhielt so einen empirischen Zusammenhang zwischen dem Atomabstand und der Magnetisierung ${ }^{9}$. Doch liegen $\mathrm{Fe}$ und $\mathrm{Ni}$ gerade umgekehrt auf dieser Néelschen Kurve, wie man es nach der bisherigen Erklärung für die Druckabhängigkeit von $\theta$ erwarten würde.

Zum Schluß möchte ich Herrn Prof. Dr. U. Dehlinger für sein reges Interesse an der vorliegenden Arbeit sowie für seine wertvollen Hinweise herzlich danken. Auch Herrn Dr. Seeger gilt mein Dank für die stete Hilfe bei der Einarbeitung in die umfangreiche Literatur sowie für viele anregende Diskussionen.

${ }^{6}$ M. Kornetzki, Physik. Z. 44, 296 [1943]; R. Bozorth, l. c. ${ }^{1}$, S. 726 .

7 J. De Boer u. A. Michels, Physica 5, 775 [1938]; J. C. Slater, Physic. Rev. 58, 54 [1940].

${ }^{8}$ R. Bozorth, l. c. ${ }^{1}$, S. 644 .

${ }^{9}$ L. Néel, Ann. Physique 8, 237 [1937].

\section{Die Initiierungsempfindlichkeit von Sekundärsprengstoffen}

Von R. Schall*

(Z. Naturforschg. 8a, 676 [1953]; eingeg. am 10. Oktober 1953)

Es wird die Minimalenergie und Leistung betrachtet, die einem Sekundärsprengkörper an einer ebenen Oberfläche zugeführt werden muß, wenn dieser unmittelbar durch einen Verdichtungsstoß zur Detonation kommen soll. Zur Erzeugung einer selbständig sich erhaltenden Detonationswelle muß der Stoß mindestens die Tiefe einer Reaktionszonenlänge $a$ und die Wellenfront einen Krümmungsradius $r>a$ haben. Bei einer Verdichtung $\varrho_{1} / \varrho_{0}=4 / 3 \mathrm{im} \mathrm{Stoß} \mathrm{-} \mathrm{wie} \mathrm{sie} \mathrm{bei} \mathrm{kon-}$ densierten Sprengstoffen näherungsweise vorliegt und unter der durch experimentelle Ergebnisse nahegelegten Annahme, daß die Länge der Reaktionszone

* Weil an Rhein. der Wellengeschwindigkeit $D$ umgekehrt proportional ist, ergibt sich die erforderliche Energie zu

$$
E=1 / 6 \varrho_{0} a_{i}{ }^{3} D_{i}{ }^{3} V^{-1},
$$

wobei $\varrho_{0}$ die Ladedichte des Sprengstoffes, $V$ die Anfangsgeschwindigkeit des zündenden Stoßes bedeutet und der Index $i$ sich auf stationäre ebene Wellen bezieht. Damit wird $E$ der abgegebenen Leistung der initiierenden Energiequelle umgekehrt proportional. Soweit vergleichbare experimentelle Werte vorliegen, ergibt sich - unter Berücksichtigung des Wirkungsgrades des Initialsprengstoffes — gute Übereinstimmung zwischen den nach (1) berechneten und bei $\mathrm{PbN}_{6}$ beobachteten Grenzladungen. Umgekehrt kann aus der Grenzladung auf die Reaktionslänge geschlossen werden.

Es liegt somit eine physikalisch befriedigende Angabe für die Empfindlichkeit von Sekundärsprengkörpern vor, die diese mit meßbaren Sprengstoffdaten in Zusammenhang bringt. Eine ausführliche Veröffentlichung erfolgt in den Nobel-Heften. 


\section{Bemerkung zu einer Arbeit von H. L. Jordan}

$$
\text { Von G. Höhler }
$$

Institut für theoretische Physik der Universität Göttingen

(Z. Naturforschg. 8a, 677 [1953]; eingeg. am 20. Oktober 1953)

In seiner Arbeit über die ,Begrenzung der Lokalisierbarkeit von Wechselwirkungen" 1 betrachtet Jordan u. a. die Spezialisierung seines Ansatzes auf den Fall, da $B$ der Integralkern $G$ im raumartigen Bereich Null ist (,,keine Überlichtgeschwindigkeit"). Er benutzt dabei eine im Anhang hergeleitete Formel, nach der die Fourier-Transformierte einer derartigen Funktion ebenfalls im ,raumartigen“ Bereich des $k$ Raumes verschwindet. Beim Beweis sind leider Vorzeichenfehler unterlaufen (Anhang, Formeln für $G^{+}$, $\left.G^{-}\right)$. Mit richtigen Vorzeichen führt die Rechnung auf ein von mir angegebenes Resultat ${ }^{2}$.

Ich danke Herrn Jordan für eine briefliche Diskussion.

${ }^{1}$ H. L. J or d a n, Z. Naturforschg. 8a, 341 [1953]. (38).

\section{BESPRECHUNGEN}

La constitution physique de la terre. ,Sciences d'aujourd'hui." Von J. Coulomb. Collection dirigée par André George, Éditions Albin Michel, 22 Rue Huyghenes, Paris 1952; 284 S., 74 Abbildungen.

Das vorliegende Buch gibt einen monographischen Überblick über die verschiedenen methodischen Wege zur Ermittlung von Erfahrungen über den physikalischen Zustand des Erdinneren und deren Ergebnisse.

Coulomb gliedert die Darstellung in vier Abschnitte: An erster Stelle steht die Behandlung der Seismologie (86 Seiten). Der zweite Abschnitt ist der gravimetrischen Problemgruppe - Schwere, Geoidform, Isostasie - gewidmet (62 Seiten). Im dritten Teil werden der Innenaufbau des Erdkörpers Schwere, Dichte, Druck in verschiedenen Tiefen seine Gezeitenerscheinungen, Polschwankungen und verwandten Probleme besprochen (50 Seiten). Das vierte Kapitel behandelt den Fragenkomplex der Temperatur und der elastischen Konstanten im Inneren und diskutiert die verschiedenen Vorstellungen über die Geschichte und den heutigen Zustand des Erdinneren (63 Seiten). Eine Zusammenstellung von 20 zusammenfassenden Darstellungen und 47 Einzelarbeiten beschließt das Buch.

Der Verfasser hat es verstanden, in geschickter Auswahl und Gruppierung des Stoffes einen lebendigen und fesselnden Überblick über die teilweise noch stark im Fluß befindlichen Vorstellungen vom Erdinneren und seinen physikalischen Eigenschaften zu entwerfen und gleichzeitig dem Leser Eingang in die Arbeitsund Denkweise und in die Problematik der Geophysik zu vermitteln.

H. Isrä̈l, Aachen.

Grundlagen der Elektroakustik. Von W. R ei c hardt. Akademische Verlagsgesellschaft Geest \& Portig, Leipzig 1952. VIII, 464 S. mit 311 Abb. und mehreren Tabellen; Preis geb. DM 32.-.

Nach einleitenden Bemerkungen über Bezeichnungen und Maßsysteme und nach einer Übersicht über die modernen Probleme der Elektroakustik werden zunächst die physikalischen Grundbegriffe des Schallfelds, wie z. B. fortschreitende und stehende Wellen, Reflexion, Beugung und Brechung, lineare und nichtlineare Schallvorgänge behandelt. Es folgen Abschnitte über das Schallempfinden des Menschen, die Schallwandler, Mikrophoneinsatz, Beschallungstechnik, elektrische Schallübertragung und Schallaufzeichnung. Ein Literaturverzeichnis und ein Sachverzeichnis schließen den Band, welcher unter Verwendung einer großen Anzahl von Abbildungen eine Einführung in die gesamte Elektroakustik gibt.

Wie der Verfasser im Vorwort betont, wird der Behandlung elektromechanischer Analogien besondere Aufmerksamkeit gewidmet. Der von der Elektrotechnik herkommende Leser wird dies begrüßen, denn ihm werden an Hand der Analogien manche akustischen Fragen leichter verständlich werden. Dem Physiker ist das Verständnis insofern nicht gerade erleichtert, als vom Verfasser vielfach ungewöhnliche, teilweise überhaupt nicht gebräuchliche Definitionen eingeführt werden. Andererseits werden viele in der akustischen Literatur seit langem fest eingeführte, bestens bewährte und sehr anschauliche Begriffe wie z. B. die mitschwingende Mediummasse und der Strahlungswiderstand von Strahlern in dem Buch nicht benutzt. Es wären zumindest Hinweise darauf notwendig gewesen, wie die neuen Vorschläge sich an die bisher gebräuchlichen Begriffe anschließen.

Es ist schwer zu vermeiden, daß in einem Buch, welches ein derart großes Gebiet mit den verschiedenartigsten Beziehungen auch zu anderen Forschungsrichtungen behandelt, manche spezielle Fragen vielleicht nicht ganz erschöpfend behandelt sind. So wird z. B. in dem Kapitel über die Trägheit des Ohres das Richtungsempfinden des Ohres ausschließlich unter dem Blickpunkt des Zeitdifferenzeffektes behandelt; der für das Richtungsempfinden bei höheren Frequenzen entscheidend wichtige Summenlokalisationseffekt wird nicht besprochen. Im übrigen ist auch im Kapitel ,Stereophonische Übertragung“ nur vom Zeitdifferenzeffekt und nicht vom Summenlokalisationseffekt die Rede. 